\begin{tabular}{|c|c|}
\hline Title & $\begin{array}{l}\text { A pproximate Scal ar Finite-Element A nalysis of A ni sotropic Optical Waveguides with Off-Diagonal Elements in a } \\
\text { Permittivity Tensor }\end{array}$ \\
\hline Author(s) & Koshiba, M.; Hayata, K.; Suzuki, M. \\
\hline Citation & IEEE Transactions on Microwave Theory and Techniques, 32(6), 587-593 \\
\hline Issue Date & $1984-01$ \\
\hline Doc URL & http:/hdl .handle.net/2115/6043 \\
\hline Rights & $\begin{array}{l}\text { C1984 IEEE. Personal use of this material is permitted. However, permission to reprint/republish this material for } \\
\text { advertising or promotional purposes or for creating new collective works for resale or redistribution to servers or lists, } \\
\text { or to reuse any copyrighted component of this work in other works must be obtained from the IEEE. } \\
\text { IEEE, IEEE Transactions on Microwave Theory and Techniques, 32(6), 1984, p587-593 }\end{array}$ \\
\hline Tyре & article \\
\hline File Information & ITMTT32_6.pdf \\
\hline
\end{tabular}

Instructions for use 


\title{
Approximate Scalar Finite-Element Analysis of Anisotropic Optical Waveguides with Off-Diagonal Elements in a Permittivity Tensor
}

\author{
MASANORI KOSHIBA, SENIOR MEMBER, IEEE, KAZUYA HAYATA, AND \\ MICHIO SUZUKI, SENIOR MEMBER, IEEE
}

Abstract - An approximate scalar finite-element program for the analysis of anisotropic optical waveguides having a permittivity tensor with nonzero off-diagonal elements is described. In this approach, the nonphysical spurious solutions which are included in the solutions of the earlier vectorial finite-element method in an axial-components formulation do not appear. Numerical examples on an anisotropic dielectric rectangular waveguide composed of a uniaxial medium are given. Our results for the waveguide whose optic axis lies in the plane ( $x y$-plane) normal to the direction ( $z$-axis) of propagation agree well with the results of the vectorial wave analysis using the variational method. We also demonstrate the application of this approach by analyzing the anisotropic dielectric rectangular waveguide whose optic axis lies in the $x z$ - or $y z$-plane.

\section{INTRODUCTION}

\section{S} EVERAL METHODS FOR the analysis of a threedimensional optical waveguide such as shown in Fig. 1 (where $t$ is the height or depth and $W$ is the width) have been proposed [1] and the vectorial finite-element method in an axial components $\left(E_{z}-H_{z}\right)$ formulation, which enables one to compute accurately the mode spectrum of a waveguide with arbitrary cross section, is widely used [2]-[8]. However, the vectorial finite-element solutions have been known to include nonphysical solutions [2]-[8]. If one wants to compute a set of eigenmodes, it is difficult and very cumbersome to distinguish between the spurious and the physical modes of the guides. The cause of these spurious modes is believed to be in the indefinite nature of the $E_{z}-H_{z}$ variational formulation [6]-[8]. In addition, the vectorial finite-element method can be applied only to anisotropic waveguides with a diagonal permittivity tensor [3], [7].

Recently, Steinberg and Giallorenzi [9] have formulated an approximate coupled mode treatment based on the Marcatili method [10] for a uniaxial waveguide whose optic axis lies in the $x z$-plane, and Ohtaka [11] has analyzed a uniaxial waveguide whose optic axis lies in the $x y$-plane using the variational method. Mabaya, Lagasse, and Vandenbulcke [7] have presented an approximate scalar

Manuscript received June 15, 1983; revised January 3, 1984.

The authors are with the Department of Electronic Engineering, Hokkaido University, Sapporo, 060, Japan.

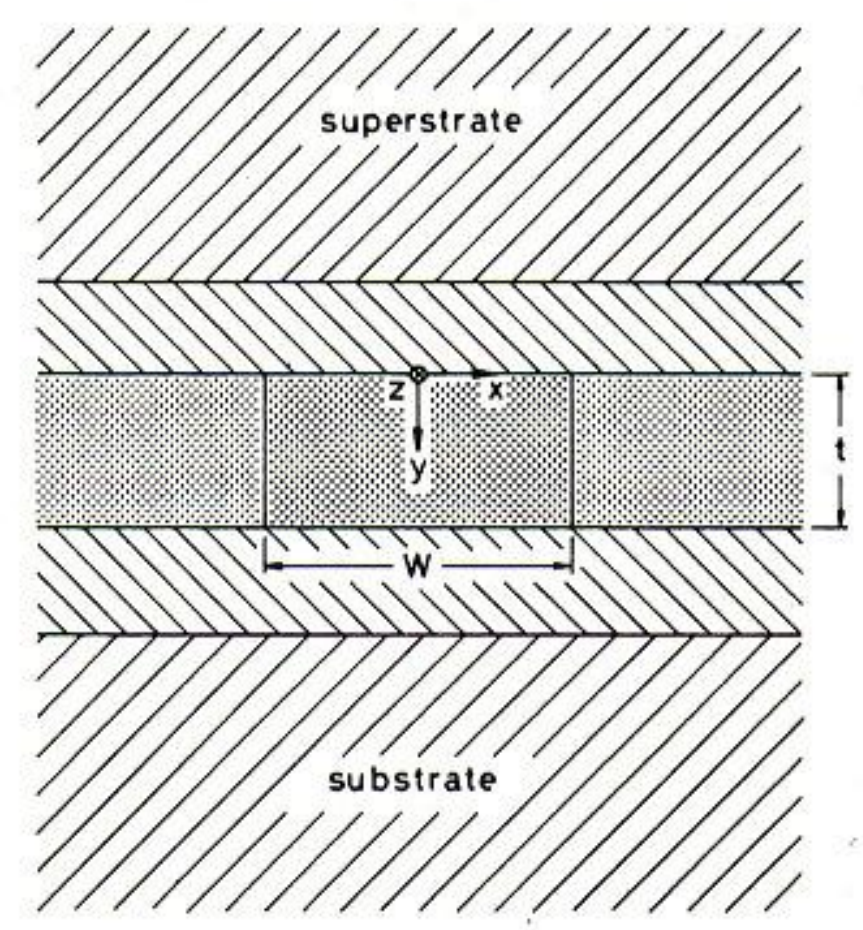

Fig. 1. Three-dimensional optical waveguide geometry.

finite-element formulation for the analysis of isotropic optical waveguides. This approach has as its main advantages: the smaller matrix dimensions, less computer time, no spurious modes (because functionals based on the scalar approximation are positive definite [7]), and the capability of easily computing higher order modes [7], [12], [13].

In this paper, this approximate scalar finite-element method is extended to the anisotropic waveguides having a permittivity tensor with nonzero off-diagonal elements. For two-dimensional waveguides $(\partial / \partial x=0)$ [14]-[17], the matrix equation derived by this approach is reduced to the exact expression for two-dimensional guided modes [18], [19]. In order to study the accuracy of the method, various isotropic dielectric waveguides are analyzed and the results obtained are compared with previously published results [5], [10], [20]. Then, numerical examples on an anisotropic dielectric rectangular waveguide composed of a uniaxial medium are given. Our results for the waveguide whose optic axis lies in the $x y$-plane agree well with the results of the vectorial wave analysis using the variational method [11]. We also demonstrate the application of this approach by analyzing the anisotropic dielectric rectangular waveguide whose optic axis lies in the $x z$ - or $y z$-plane. 


\section{APProximate Basic Equations}

We consider a three-dimensional anisotropic waveguide in Fig. 1 having a permittivity tensor with all nonzero off-diagonal elements $\epsilon_{0} K_{i j}(i, j=x, y, z)$ and the permeability of free space $\mu_{0}$, where $\epsilon_{0}$ is the permittivity of free space and $K_{i j}$ is the relative permittivity.

Maxwell's equations are, in component form

$$
\begin{gathered}
\partial E_{z} / \partial y+j k_{z} E_{y}=-j \omega \mu_{0} H_{x} \\
-j k_{z} E_{x}-\partial E_{z} / \partial x=-j \omega \mu_{0} H_{y} \\
\partial E_{y} / \partial x-\partial E_{x} / \partial y=-j \omega \mu_{0} H_{z} \\
\partial H_{z} / \partial y+j k_{z} H_{y}=j \omega D_{x} \\
-j k_{z} H_{x}-\partial H_{z} / \partial x=j \omega D_{y} \\
\partial H_{y} / \partial x-\partial H_{x} / \partial y=j \omega D_{z} \\
H_{z}=\left(1 / j k_{z}\right)\left(\partial H_{x} / \partial x+\partial H_{y} / \partial y\right) \\
D_{z}=\left(1 / j k_{z}\right)\left(\partial D_{x} / \partial x+\partial D_{y} / \partial y\right)
\end{gathered}
$$

where $E_{i}, H_{i}$, and $D_{i}$ are the electric field, the magnetic field, and the electric flux density, respectively, and $\omega$ and $k_{z}$ are the angular frequency and the wavenumber in the $z$-direction, respectively.

The constitutive relations are

$$
\begin{aligned}
& D_{x}=\epsilon_{0}\left(K_{x x} E_{x}+K_{x y} E_{y}+K_{x z} E_{z}\right) \\
& D_{y}=\epsilon_{0}\left(K_{x y} E_{x}+K_{y y} E_{y}+K_{y z} E_{z}\right) \\
& D_{z}=\epsilon_{0}\left(K_{x z} E_{x}+K_{y z} E_{y}+K_{z z} E_{z}\right) .
\end{aligned}
$$

We assume $\left|K_{i j}\right| \ll K_{i i}(i \neq j)$ and a small index variation in the lateral $(x)$ direction, then we may approximate as

$$
K_{i j} \partial / \partial x \doteqdot 0, \quad i \neq j .
$$

Considering (12), from (2) and (11) we obtain

$$
H_{y}=\frac{1}{j \omega \mu_{0}}\left(j k_{z} E_{x}+\frac{1}{\epsilon_{0} K_{z z}} \frac{\partial D_{z}}{\partial x}\right) .
$$

Substituting (8) into (13) and considering (9), (10), and (12), we obtain

$H_{y}=\frac{1}{j \omega \mu_{0}}\left[j k_{z} E_{x}+\frac{1}{j k_{z} K_{z z}} \frac{\partial}{\partial x}\left(K_{x x} \frac{\partial E_{x}}{\partial x}+K_{y y} \frac{\partial E_{y}}{\partial y}\right)\right]$.

We write (3)

$$
H_{z}=\frac{1}{j \omega \mu_{0}}\left(\frac{\partial E_{x}}{\partial y}-\frac{\partial E_{y}}{\partial x}\right) .
$$

Considering (10)-(12) and eliminating $E_{z}$ between (5) and (6), we obtain

$E_{y}=-\frac{1}{j \omega \epsilon_{0}} \frac{1}{a}\left(j k_{z} K_{z z} H_{x}+K_{z z} \frac{\partial H_{z}}{\partial x}-K_{y z} \frac{\partial H_{x}}{\partial y}\right)-\frac{b}{a} E_{x}$.
Substituting (7) into (16), we obtain

$$
\begin{aligned}
E_{y}=-\frac{1}{j \omega \epsilon_{0}} \frac{1}{a}\left[j k_{z} K_{z z} H_{x}+\right. & \frac{K_{z z}}{j k_{z}} \frac{\partial}{\partial x}\left(\frac{\partial H_{x}}{\partial x}+\frac{\partial H_{y}}{\partial y}\right) \\
& \left.-K_{y z} \frac{\partial H_{x}}{\partial y}\right]-\frac{b}{a} E_{x} .
\end{aligned}
$$

Considering (10)-(12) and eliminating $E_{y}$ between (5) and (6), we obtain

$$
E_{z}=-\frac{1}{j \omega \epsilon_{0}} \frac{1}{a}\left(K_{y y} \frac{\partial H_{x}}{\partial y}-K_{y y} \frac{\partial H_{y}}{\partial x}-j k_{z} K_{y z} H_{x}\right)-\frac{c}{a} E_{x}
$$

where

$$
\begin{aligned}
& a=K_{y y} K_{z z}-K_{y z}^{2} \\
& b=K_{x y} K_{z z}-K_{x z} K_{y z} \\
& c=K_{x z} K_{y y}-K_{x y} K_{y z} .
\end{aligned}
$$

Substituting (14), (15), (17), and (18) into (4), considering (12) and neglecting the terms of $E_{y}$ in the same manner as in the isotropic case [7], [12], we obtain

$$
\begin{aligned}
\frac{K_{x x}}{K_{z z}} \frac{\partial}{\partial x}\left(\frac{\partial \phi}{\partial x}\right) & +\frac{\partial}{\partial y}\left(\frac{\partial \phi}{\partial y}\right) \\
& +\left[k_{0}^{2}\left(K_{x x}-K_{x y} \frac{b}{a}-K_{x z} \frac{c}{a}\right)-k_{z}^{2}\right] \phi \\
& -k_{0} k_{z} \frac{b}{a} \psi+j k_{0} \frac{c}{a} \frac{\partial \psi}{\partial y}=0 .
\end{aligned}
$$

Similarly, substituting (17) and (18) into (1) and neglecting the terms of $H_{y}$ in the same manner as in the isotropic case [7], [12], we obtain

$$
\begin{array}{r}
\frac{K_{z z}}{a} \frac{\partial}{\partial x}\left(\frac{\partial \psi}{\partial x}\right)+\frac{\partial}{\partial y}\left(\frac{K_{y y}}{a} \frac{\partial \psi}{\partial y}-j k_{z} \frac{K_{y z}}{a} \psi+j k_{0} \frac{c}{a} \phi\right) \\
+\left(k_{0}^{2}-k_{z}^{2} \frac{K_{z z}}{a}\right) \psi-j k_{z} \frac{K_{y z}}{a} \frac{\partial \psi}{\partial y}-k_{0} k_{z} \frac{b}{a} \phi=0
\end{array}
$$

where

$$
\begin{aligned}
\phi & =E_{x} \\
\psi & =\eta_{0} H_{x} \\
k_{0} & =\omega \sqrt{\epsilon_{0} \mu_{0}} \\
\eta_{0} & =\sqrt{\mu_{0} / \epsilon_{0}} .
\end{aligned}
$$

If the waveguide is isotropic, namely $K_{x x}=K_{y y}=K_{z z} \equiv$ $n^{2}$ and $K_{x y}=K_{x z}=K_{y z}=0,(20)$ is reduced to the Helmholtz equation for the $\mathrm{TE}_{y}$ mode $\left(E_{y} \equiv 0\right)$ and $(21)$ is reduced to that for the $\mathrm{TM}_{y}$ mode $\left(H_{y} \equiv 0\right)$ derived by Yasuura, Shimohara, and Miyamoto [20], where $n$ is the refractive index. In the case of an infinite slab waveguide ( $\partial / \partial x=0$, two-dimensional waveguide) [14]-[17], the approximate basic equations (20) and (21) are reduced to the exact equations for the two-dimensional guided modes.

\section{FINITE-ELEMENT APPROACH}

The finite-element formulation is based on the following variational expression [2], [3], [5]-[8], [18] for previous 


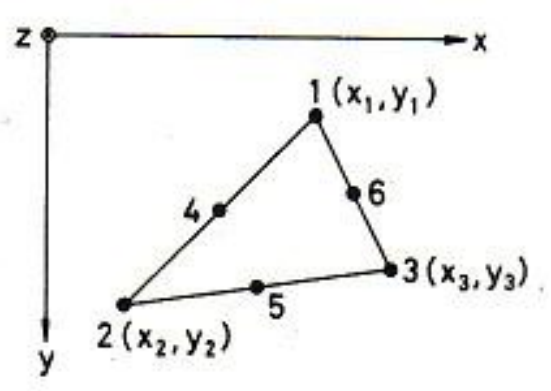

Fig. 2. 2nd-order triangular element.

equations (20) and (21):

$$
\delta I=0
$$

where

$$
\begin{aligned}
I= & \iint_{\Omega}\left(\frac{K_{x x}}{K_{z z}} \frac{\partial \phi^{*}}{\partial x} \frac{\partial \phi}{\partial x}+\frac{\partial \phi^{*}}{\partial y} \frac{\partial \phi}{\partial y}\right. \\
& +\left[k_{z}^{2}-k_{0}^{2}\left(K_{x x}-K_{x y} \frac{b}{a}-K_{x z} \frac{c}{a}\right)\right] \phi^{*} \phi \\
& +\frac{K_{z z}}{a} \frac{\partial \psi^{*}}{\partial x} \frac{\partial \psi}{\partial x}+\frac{K_{y y}}{a} \frac{\partial \psi^{*}}{\partial y} \frac{\partial \psi}{\partial y}+\left(k_{z}^{2} \frac{K_{z z}}{a}-k_{0}^{2}\right) \psi^{*} \psi \\
& +j k_{z} \frac{K_{y z}}{a}\left(\psi^{*} \frac{\partial \psi}{\partial y}-\frac{\partial \psi^{*}}{\partial y} \psi\right) \\
& +k_{0} k_{z} \frac{b}{a}\left(\phi^{*} \psi+\psi^{*} \phi\right) \\
& \left.-j k_{0} \frac{c}{a}\left(\phi^{*} \frac{\partial \psi}{\partial y}-\frac{\partial \psi^{*}}{\partial y} \phi\right)\right) d x d y
\end{aligned}
$$

Here, $\Omega$ is the cross section of the guide and the asterisk denotes complex conjugate.

Dividing the cross section of the guide into a number of 2nd-order triangular elements as shown in Fig. 2, $\phi$ in (22) and $\psi$ in (23) within each element are defined in terms of $\phi_{k}$ and $\psi_{k}$ at the nodal points $k(k=1,2, \cdots, 6)$, respectively, as follows:

$$
\begin{aligned}
& \phi=\{N\}^{T}\{\phi\}_{e} \\
& \psi=\{N\}^{T}\{\psi\}_{e}
\end{aligned}
$$

where

$$
\begin{aligned}
& \{\phi\}_{e}=\left[\phi_{1} \phi_{2} \phi_{3} \phi_{4} \phi_{5} \phi_{6}\right]^{T} \\
& \{\psi\}_{e}=\left[\psi_{1} \psi_{2} \psi_{3} \psi_{4} \psi_{5} \psi_{6}\right]^{T} \\
& \{N\}=\left[N_{1} N_{2} N_{3} N_{4} N_{5} N_{6}\right]^{T} .
\end{aligned}
$$

Here $T,\{\cdot\}$, and $\{\cdot\}^{T}$ denote a transpose, a column vector, and a row vector, respectively, and the shape functions $N_{1}$ to $N_{6}$ are given by

$$
\begin{aligned}
& N_{1}=L_{1}\left(2 L_{1}-1\right) \\
& N_{2}=L_{2}\left(2 L_{2}-1\right) \\
& N_{3}=L_{3}\left(2 L_{3}-1\right) \\
& N_{4}=4 L_{1} L_{2} \\
& N_{5}=4 L_{2} L_{3} \\
& N_{6}=4 L_{3} L_{1}
\end{aligned}
$$

with the area coordinates $L_{1}, L_{2}$, and $L_{3}$ [2], [5]. The relation equation between the area coordinates and Carte- sian coordinates is given by

$$
\left[\begin{array}{l}
x \\
y \\
1
\end{array}\right]=\left[\begin{array}{ccc}
x_{1} & x_{2} & x_{3} \\
y_{1} & y_{2} & y_{3} \\
1 & 1 & 1
\end{array}\right]\left[\begin{array}{l}
L_{1} \\
L_{2} \\
L_{3}
\end{array}\right]
$$

where $\left(x_{l}, y_{l}\right)$ are the Cartesian coordinates of the vertex $l$ $(l=1,2,3)$ of the triangle.

Both $\phi$ and $\psi$ fields exist in the medium (or media) surrounding the optical waveguide, and these fields extend to infinity. One method for modeling the surround is imposing an artificial zero boundary condition for $\phi$ and $\psi$ at a large enough distance from the guide. This method has as an advantage its simplicity and is widely used [2]-[8], [12], [13]. Using this zero boundary condition and substituting (28) and (29) into (27), from (26) we obtain the following global matrix equation:

where

$$
\left[\begin{array}{ll}
{[A]} & {[B]} \\
{[C]} & {[D]}
\end{array}\right]\left[\begin{array}{l}
\{\phi\} \\
\{\psi\}
\end{array}\right]=\{0\}
$$

$$
\begin{aligned}
{[A]=} & \sum_{e} \iint_{\Omega_{e}}\left(\frac{K_{x x, e}}{K_{z z, e}} \frac{\partial\{N\}}{\partial x} \frac{\partial\{N\}^{T}}{\partial x}+\frac{\partial\{N\}}{\partial y} \frac{\partial\{N\}^{T}}{\partial y}\right. \\
& -\left[k_{0}^{2}\left(K_{x x, e}-K_{x y, e} \frac{b_{e}}{a_{e}}-K_{x z, e} \frac{c_{e}}{a_{e}}\right)-k_{z}^{2}\right] \\
& \left.\cdot\{N\}\{N\}^{T}\right) d x d y
\end{aligned}
$$

$$
\begin{aligned}
{[B]=\sum_{e} \iint_{\Omega_{e}}\left(k_{0} k_{z}\right.} & \frac{b_{e}}{a_{e}}\{N\}\{N\}^{T} \\
& \left.-j k_{0} \frac{c_{e}}{a_{e}}\{N\} \frac{\partial\{N\}^{T}}{\partial y}\right) d x d y
\end{aligned}
$$

$$
\begin{aligned}
{[C]=\sum_{e} \iint_{\Omega_{e}}\left(k_{0} k_{z}\right.} & \frac{b_{e}}{a_{e}}\{N\}\{N\}^{T} \\
& \left.+j k_{0} \frac{c_{e}}{a_{e}} \frac{\partial\{N\}}{\partial y}\{N\}^{T}\right) d x d y
\end{aligned}
$$

$$
\begin{aligned}
{[D]=\sum_{e} } & \iint_{\Omega_{e}}\left(\frac{K_{z z, e}}{a_{e}} \frac{\partial\{N\}}{\partial x} \frac{\partial\{N\}^{T}}{\partial x}\right. \\
& +\frac{K_{y y, e}}{a_{e}} \frac{\partial\{N\}}{\partial y} \frac{\partial\{N\}^{T}}{\partial y} \\
& +j k_{z} \frac{K_{y z, e}}{a_{e}}\left[\{N\} \frac{\partial\{N\}^{T}}{\partial y}-\frac{\partial\{N\}}{\partial y}\{N\}^{T}\right] \\
& \left.-\left[k_{0}^{2}-k_{z}^{2} \frac{K_{z z, e}}{a_{e}}\right]\{N\}\{N\}^{T}\right) d x d y .
\end{aligned}
$$

Here the summation $\Sigma_{e}$ extends over all different elements, the components of $\{\phi\}$ and $\{\psi\}$ vectors are the values of $E_{x}$ and $\eta_{0} H_{x}$ at nodal points in $\Omega$, respectively, and $\{0\}$ is a null vector.

For an anisotropic waveguide with a diagonal permittivity tensor, namely $K_{x y}=K_{x z}=K_{y z}=0$, (35) is reduced to the equations derived by Koshiba, Hayata, and Suzuki [12]. In this case, $[B]=[C]=[0]$ (where $[0]$ is a zero matrix) and 
equations $|[A]|=0$ and $|[B]|=0$ determine the dispersion characteristics for the $E_{p q}^{x}$ and $E_{p q}^{y}$ modes [10] in the waveguides with a diagonal permittivity tensor, respectively. The main field components of the $E_{p q}^{x}$ modes are $E_{x}$ and $H_{y}$, while those of the $E_{p q}^{y}$ modes are $H_{x}$ and $E_{y}$ [10]. The subscripts $p$ and $q$ are used to designate the number of maxima of the dominant field in the $x$ - and $y$-directions, respectively. In the case of a two-dimensional waveguide $(\partial / \partial x=0),(35)$ gives the exact expression for twodimensional guided modes [18], [19].

In order that a nontrivial solution of (35) may exist

$$
\left|\begin{array}{ll}
{[A]} & {[B]} \\
{[C]} & {[D]}
\end{array}\right|=0
$$

must hold. This equation is the eigenvalue (dispersion) equation which determines the dispersion characteristics for the guided modes in anisotropic waveguides having a permittivity tensor with nonzero off-diagonal elements. In the present analysis, the Cholesky method, the Householder's method, the method of bisections, and the QR method are suitably used for solving (37).

\section{Computed Results}

Equation (12) and neglect of $E_{y}$ and $H_{y}$ in (20) and (21) may not be valid for the neighborhood of cutoff frequency and for the higher order modes. Therefore, it is necessary to study the accuracy of the method.

First we consider isotropic dielectric waveguides which are analyzed by many researchers using a variety of methods [1]-[13], [20]. Fig. 3 shows the dispersion characteristics for the fundamental $E_{1}^{y}$ mode in an embossed waveguide and in a channel waveguide, where the index variation in the lateral direction of the channel waveguide is smaller than that of the embossed waveguide and the finite-element model uses 84 elements and 195 nodal points in one-half of the cross section. For the embossed waveguide, our results (solid lines) deviate from the results (dots) of the vectorial finite-element method [5] in the neighborhood of cutoff frequency when the width $W / t$ is narrow $(W / t=1$ and 2). On the other hand, for the channel waveguide with $W / t=2$, agreement between our results (dashed line) and the results (circles) of the vectorial finite-element method is good over a wide range of frequencies. In [12], the approximate scalar finite-element method is used for the analysis of an anisotropic channel waveguide with a diagonal permittivity tensor and a 1-percent variation of the ordinary and extraordinary refractive indices of $\mathrm{LiNbO}_{3}$, and the results obtained agree very well with the results of the vectorial finite-element method [3].

Fig. 4(a) shows the dispersion characteristics for the $E_{p q}^{x}$ and $E_{p q}^{y}$ modes in a rib waveguide, where the finite-element model employs 180 elements and 399 nodal points in one-half of the cross section. The $E_{11}^{x}$ (or $E_{11}^{y}$ ) and $E_{21}^{x}$ (or $\left.E_{21}^{y}\right)$ modes are the lowest modes of the symmetric ( $p$ is odd) and antisymmetric ( $p$ is even) $E_{p q}^{x}$ (or $E_{p q}^{y}$ ) modes, respectively. Our results for these modes agree well with the results of the mode-matching method [20]. However, our results for the higher order $E_{31}^{x}$ and $E_{31}^{y}$ modes are not in good agreement with the results of the mode-matching

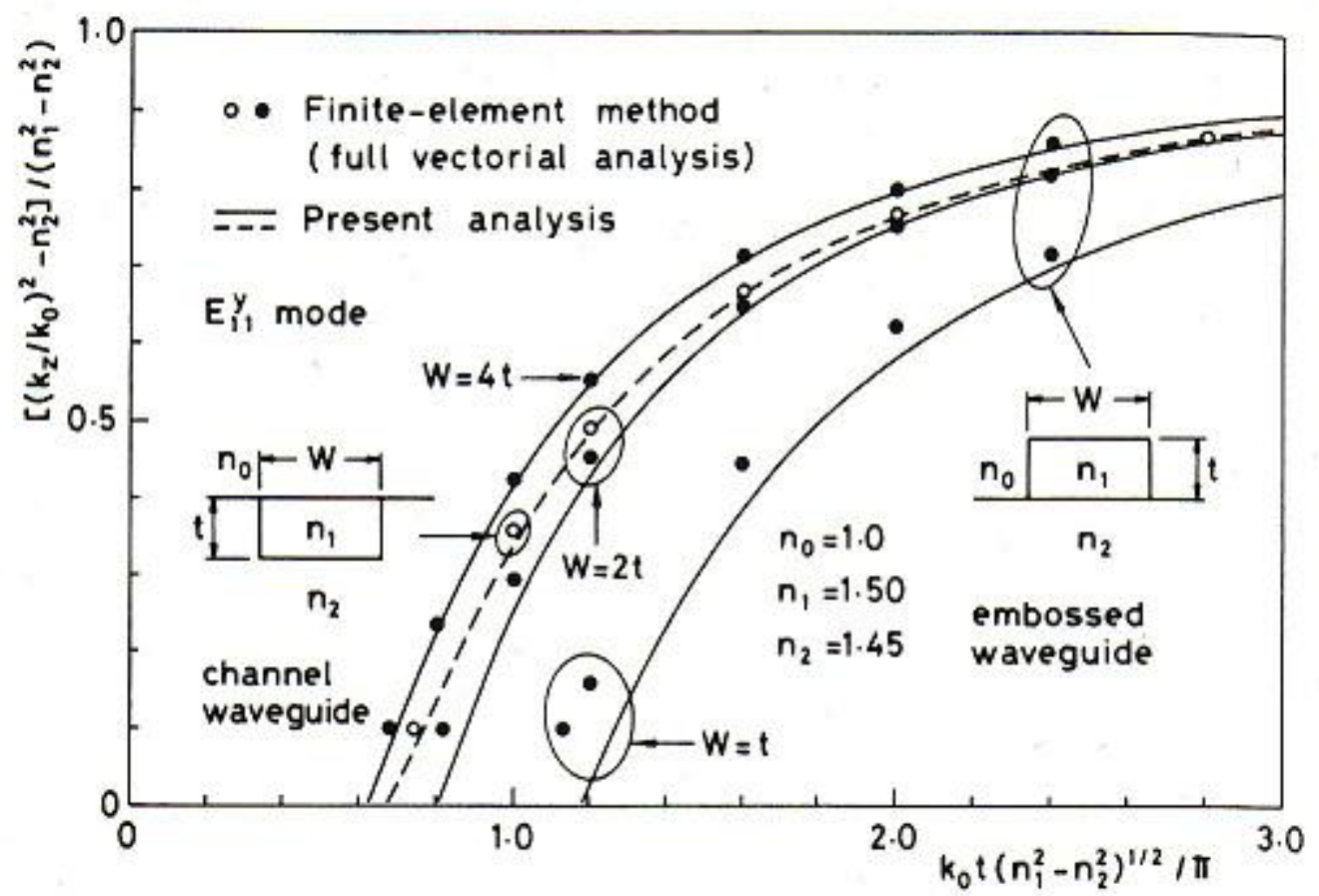

Fig. 3. Dispersion characteristics for the embossed waveguide and the channel waveguide.

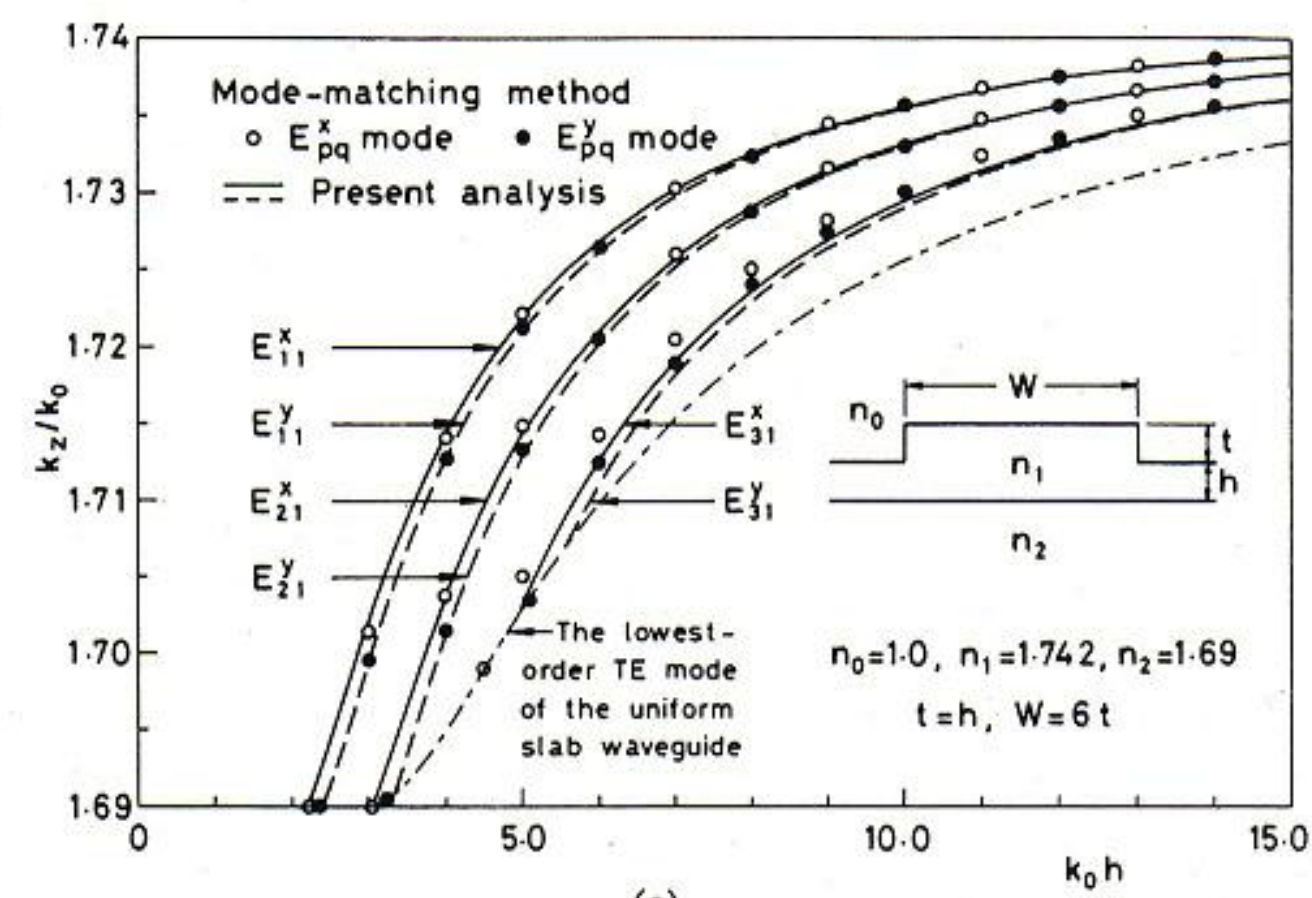

(a)

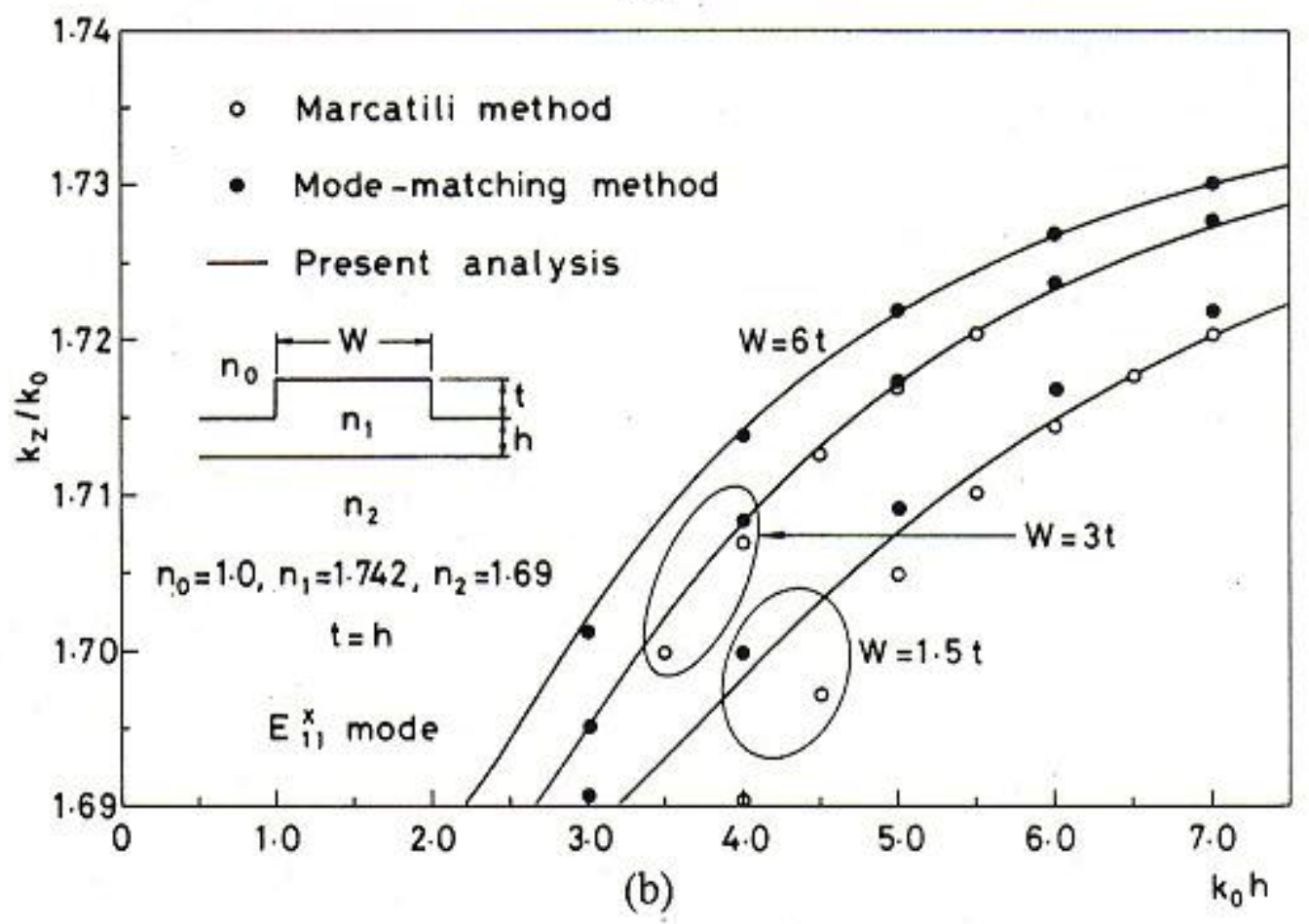

Fig. 4. Dispersion characteristics for the rib waveguide. (a) $E_{p q}^{x}, E_{p q}^{y}$ modes. (b) $E_{11}^{x}$ mode.

method, especially near the cutoff frequencies. Fig. 4(b) shows the dispersion characteristics for the fundamental $E_{11}^{x}$ mode in the rib waveguide when the width $W / t$ is altered. When the width $W / t$ becomes narrow, our results deviate from the results (dots) of the mode-matching method. But our results are closer to the results of the mode-matching method than those (circles) of Marcatili's approximate analytical approach [10]. From Figs. 3 and 4, we may conclude that the approximate scalar finite-element results are more accurate for the lower order modes in the waveguide of a small index variation in the lateral 


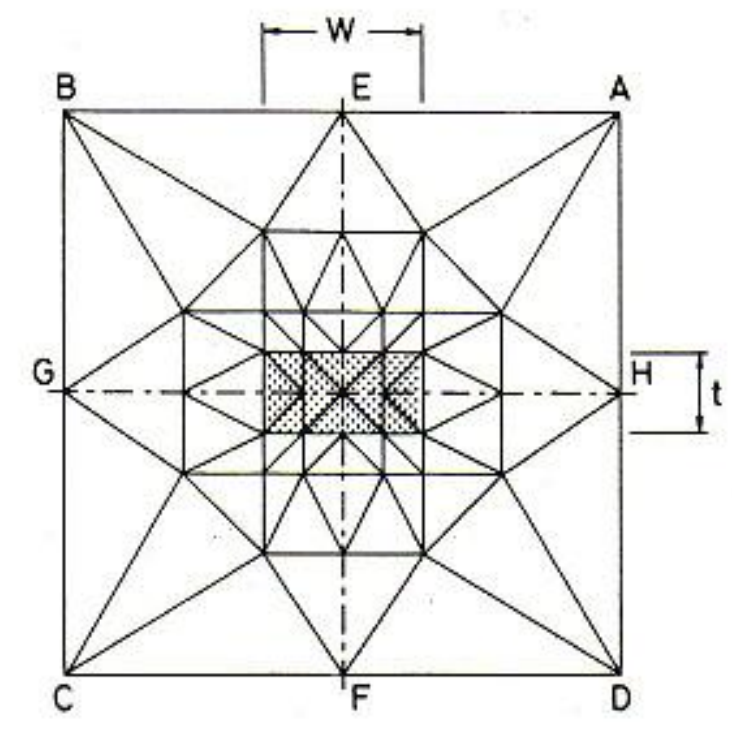

Fig. 5. Element division for the anisotropic dielectric rectangular wave guide.

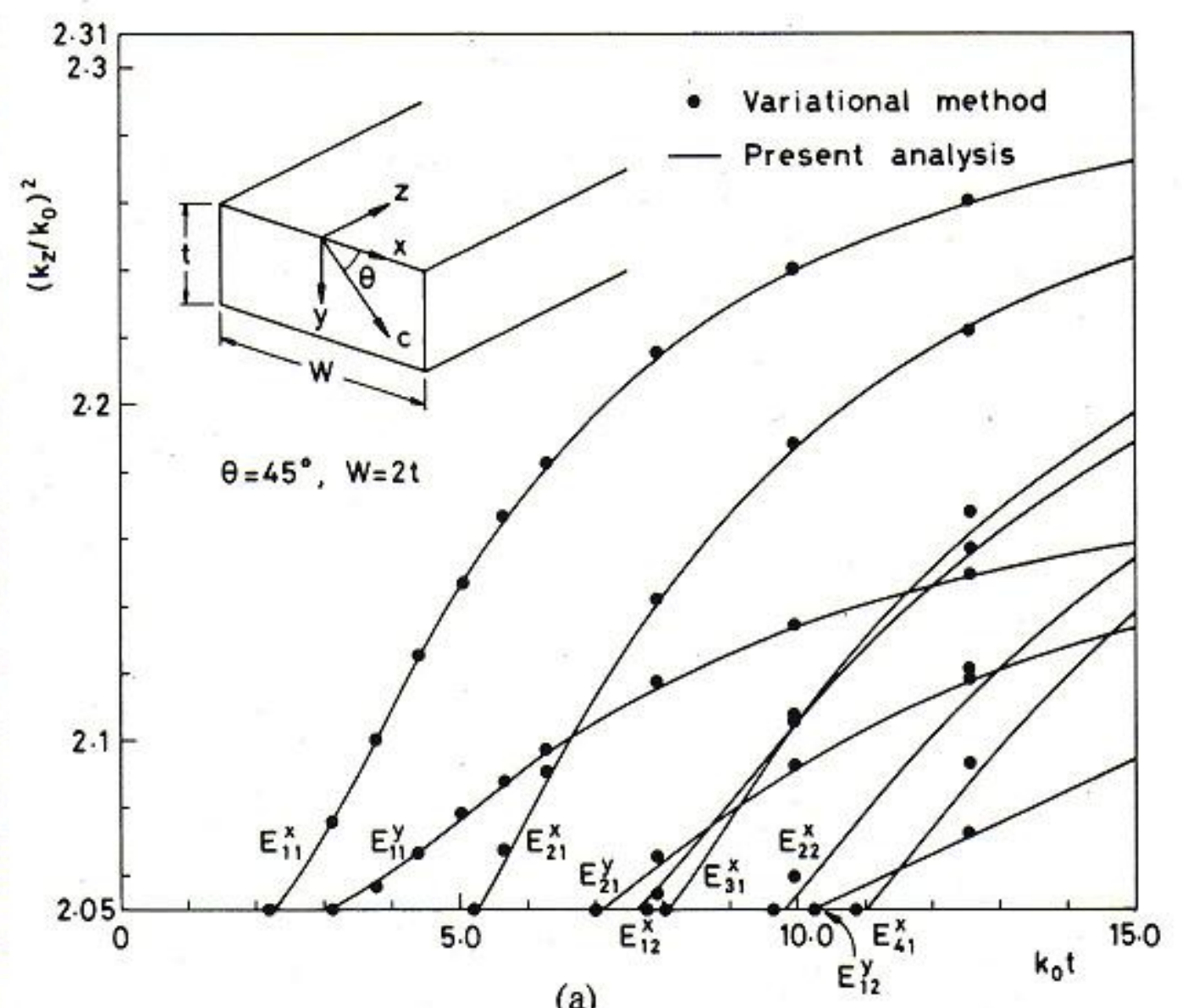

(a)

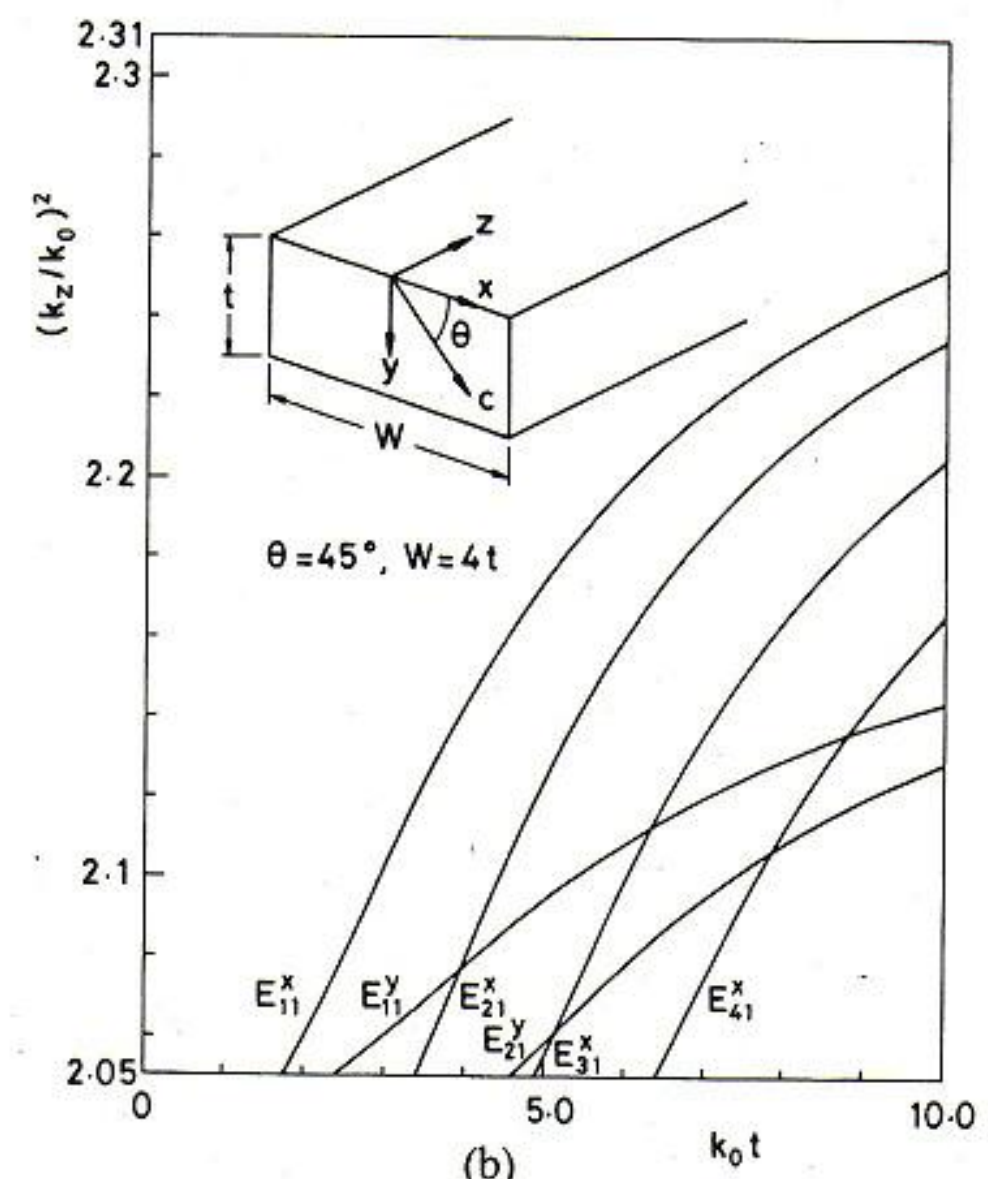

(b)

Fig. 6. Dispersion characteristics for the anisotropic dielectric rectangular waveguide whose optic axis lies in the $x y$-plane. (a) $W=2 t$. (b) $W=4 t$.

(x) direction or for those in the waveguide of a large width-to-height ratio. However, for the waveguide of a small width-to-height ratio and near the cutoff frequencies, the accuracy of the method is questionable. It seems that

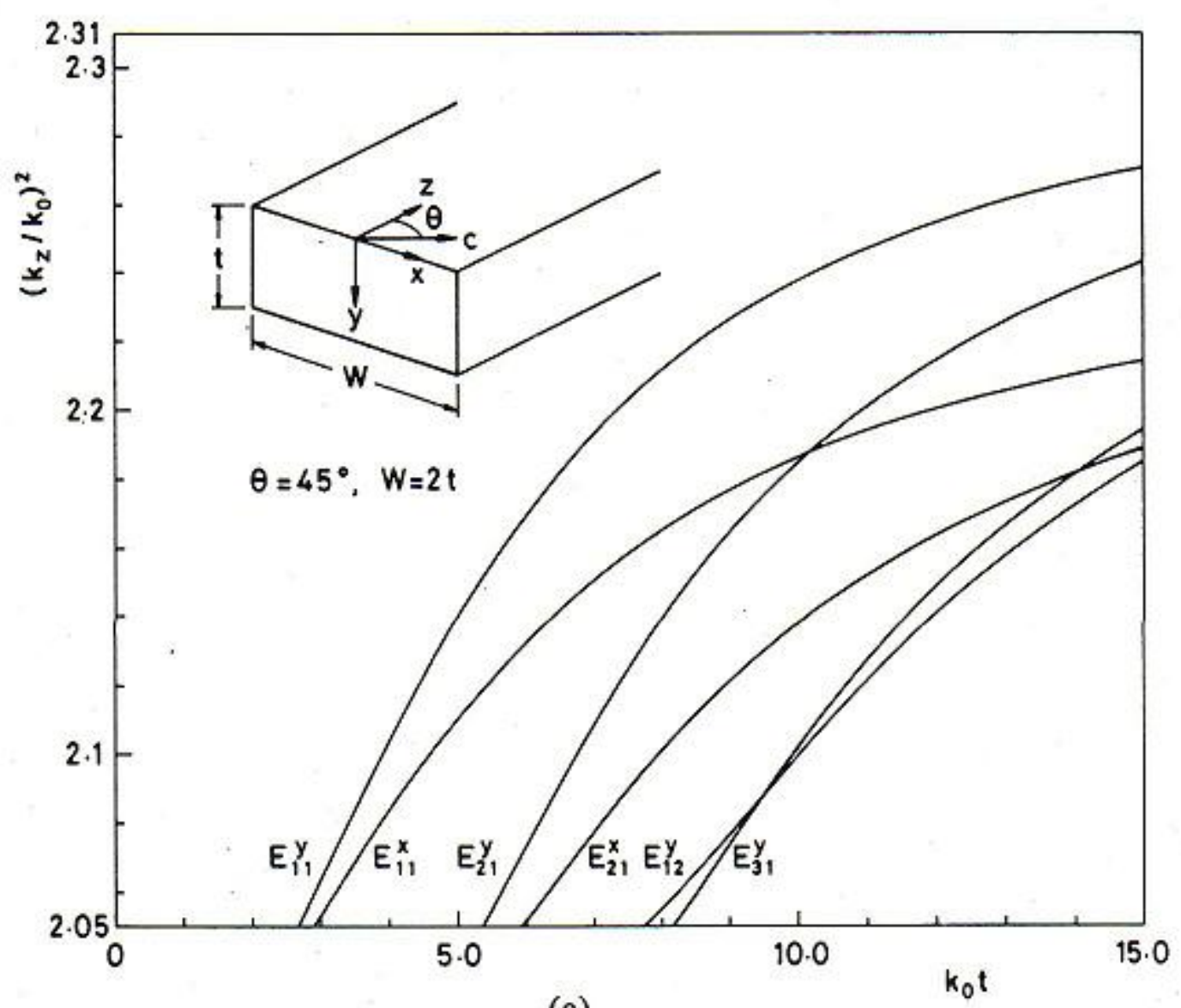

(a)

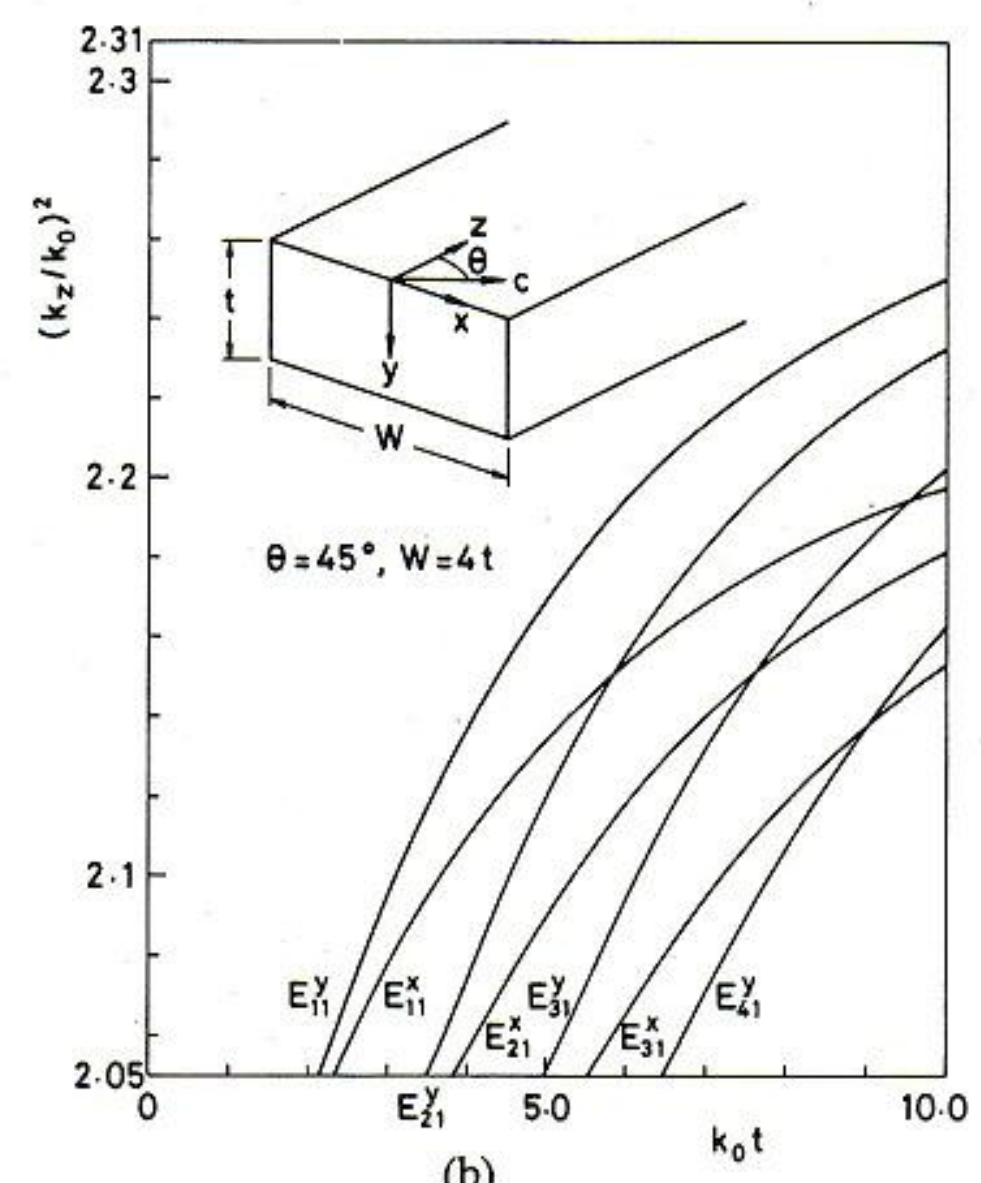

(b)

Fig. 7. Dispersion characteristics for the anisotropic dielectric rectangular waveguide whose optic axis lies in the $x z$-plane. (a) $W=2 t$. (b) $W=4 t$.

variations in accuracy are due to the scalar approximations.

As for the second example, we consider an anisotropic dielectric rectangular waveguide composed of a uniaxial medium. A typical division of this waveguide into 2 nd-order triangular elements is shown in Fig. 5, where the number of elements and of nodal points is 80 and 169 , respectively. Figs. 6-8 show the dispersion characteristics for the guided modes in the anisotropic dielectric rectangular waveguides surrounded by an isotropic medium of refractive index $\sqrt{2.05}$, where the ordinary and extraordinary refractive indices of a rectangular core are $\sqrt{2.31}$ and $\sqrt{2.19}$, respectively. The optic axis $c$ in Figs. 6, 7, and 8 lies in the $x y$-plane at an angle $\theta=45^{\circ}$ from the $x$-axis ( $K_{x y} \neq 0, K_{x z}$ $=K_{y z}=0$ ), in the $x z$-plane at an angle $\theta=45^{\circ}$ from the $z$-axis $\left(K_{x z} \neq 0, K_{x y}=K_{y z}=0\right)$, and in the $y z$-plane at an angle $\theta=45^{\circ}$ from the $z$-axis $\left(K_{y z} \neq 0, K_{x y}=K_{x z}=0\right)$, respectively. Modes are designated as $E_{p q}^{x}$ ( $E_{p q}^{x}$-like mode) 
[10] E. A. Marcatili, "Dielectric rectangular waveguide and directional coupler for integrated optics," Bell. Syst. Tech. J., vol. 48, pp. 2071-2102, Sept. 1969.

[11] M. Ohtaka, "Analysis of the guided modes in the anisotropic dielectric optical waveguides," Trans. Inst. Electron. Commun. Eng. Japan, vol. J64-C, pp.674-681, Oct. 1981.

[12] M. Koshiba, K. Hayata, and M. Suzuki, "Approximate scalar finite-element analysis of anisotropic optical waveguides," Electron. Lett., vol. 18, pp. 411-413, May 1982.

[13] M. Koshiba, K. Hayata, and M. Suzuki, "sc On accuracy of approximate scalar finite-element analysis of dielectric optical waveguides," Trans. Inst. Electron. Commun. Eng. Japan, vol. E66, pp. 157-158, Feb. 1983.

[14] S. Yamamoto, Y. Koyamada, and T. Makimoto, "Normal-mode analysis of anisotropic and gyrotropic thin-film optical waveguides and their applications," Trans. Inst. Electron. Commun. Eng. Japan, vol. 55-C, pp. 550-557, Oct. 1972.

[15] Y. Satomura, M. Matsuhara, and N. Kumagai, "Analysis of electromagnetic-wave modes in anisotropic slab waveguides," IEEE Trans. Microwave Theory Tech., vol. MTT-22, pp. 86-92, Feb. 1974.

[16] D. Marcuse, "Modes of a symmetric slab waveguides in birefringent media-Part I: Optical axis not in plane of slab," IEEE J. Quantum Electron., vol. QE-14, pp. 736-741, Oct. 1978.

[17] D. Marcuse and I. P. Kaminow, "Modes of a symmetric slab optical waveguides in birefringent media, Part II: Slab with coplanar optical axis," IEEE J. Quantum Electron., vol. QE-15, pp. 92-101, Feb. 1979.

[18] M. Hano and K. Kayano, "An analysis of anisotropic and inhomogeneous optical waveguide using finite element method," Trans. Inst. Electr. Eng. Japan, vol. 101(C), pp. 213-219, Sept. 1981.

[19] M. Koshiba and M. Suzuki, "Numerical analysis of planar arbitrarily anisotropic diffused optical waveguides using finite-element method," Electron. Lett., vol. 18, pp. 579-581, June 1982.

[20] K. Yasuura, K. Shimohara, and T. Miyamoto, "Numerical analysis of a thin-film waveguide by mode-matching method," J. Opt. Soc. Am., vol. 70, pp. 183-191, Feb. 1980.

Masanori Koshiba (SM'84) was born in Sapporo, Japan, on November 23 , 1948, He received the B.S., M.S., and Ph.D. degrees in electronic engineering from Hokkaido University, Sapporo, Japan, in 1971, 1973, and 1976 , respectively.

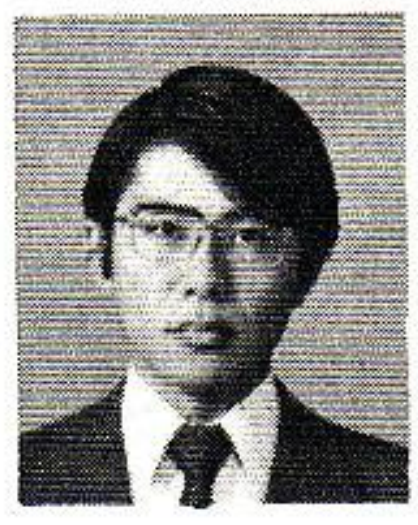

In 1976, he joined the Department of Electronic Engineering, Kitami Institute of Technology, Kitami, Japan. Since 1979, he has been an Assistant Professor of Electronic Engineering at Hokkaido University. He has been engaged in research on surface acoustics waves, dielectric optical waveguides, and applications of finite-element and boundary element methods to fields problems.

Dr. Koshiba is a member of the Institute of Electronics and Communication Engineers of Japan.

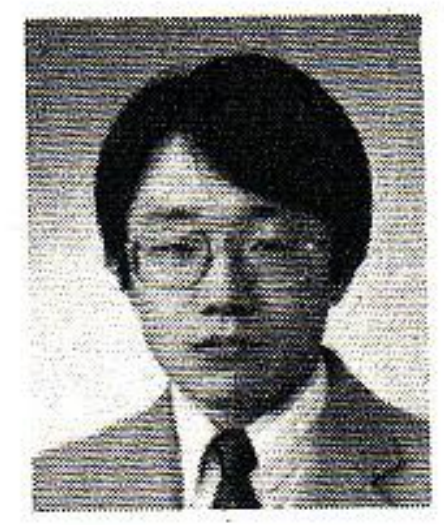

Kazuya Hayata was born in Kushiro, Japan, on December 1, 1959. He received the B.S. degree in electronic engineering from Hokkaido University, Sapporo, Japan, in 1982.

He is presently studying toward the M.S. degree in electronic engineering, Hokkaido University, Sapporo, Japan.

Mr. Hayata is a member of the Institute of Electronics and Communication Engineers of Japan.

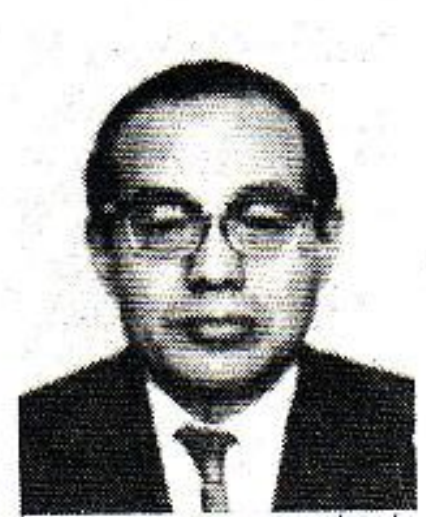

Michio Suzuki (SM'57) was born in Sapporo, Japan, on November 14, 1923. He received the B.S. and $\mathrm{Ph} . \mathrm{D}$. degrees in electrical engineering from Hokkaido University, Sapporo, Japan, in 1946 and 1960 , respectively.

From 1948 to 1962, he was an Assistant Professor of Electrical Engineering at Hokkaido University. Since 1962, he has been a Professor of Electronic Engineering at Hokkaido University. From 1956 to 1957 , he was a Research Associate at the Microwave Research Institute of the Polytechnic Institute of Brooklyn, Brooklyn, NY.

Dr. Suzuki is a member of the Institute of Electronics and Communication Engineers of Japan, the Institute of Electrical Engineers of Japan, and the Institute of Television Engineers of Japan. 